\title{
The Reliability of the 300-Yard Shuttle Run in High School Girls Basketball Players
}

\author{
Kali White ${ }^{1}$, Mark DeBeliso ${ }^{1}$, Trish Sevene ${ }^{2}$ and Kent Adams ${ }^{2}$ \\ 1. Department of Physical Education and Human Performance, Southern Utah University, Cedar City, UT 84720, USA \\ 2. Kinesiology Department, California State University Monterey Bay, Seaside, CA 93955, USA
}

\begin{abstract}
The 300-yard shuttle run is a field test commonly used to assess anaerobic capacity. There is limited documentation regarding the reliability of the shuttle run for various populations. The purpose of this study was to assess the reliability of the 300 -yard shuttle run in High School girls basketball players. Thirty nine High School girls basketball players performed two separate sessions of the 300-yard shuttle run. During each session, the participants completed two trials of the 300-yard shuttle run separated by a 5-minute rest period. The trial 1 and 2 scores were $66.2 \pm 4.9$ and $69.1 \pm 6.0$ seconds, respectively. The interclass and intraclass reliability coefficients were $\mathrm{r}=0.84$ and $\mathrm{ICC}=0.83$. The standard error of the measure was $\mathrm{SE}_{\mathrm{m}}=2.2$ seconds with $90 \%$ confidence limits of $\mathrm{U}_{\mathrm{L}}$ : 2.6, $\mathrm{L}_{\mathrm{L}}: 1.9$. The typical error expressed as a coefficient of variation percent was $\mathrm{CV} \%=3.2$ with $90 \%$ confidence limits of $\mathrm{U}_{\mathrm{L}}: 3.7, \mathrm{~L}_{\mathrm{L}}$ : 2.8. Within the parameters of this study, the 300 -yard shuttle run is a sufficiently reliable field test for measuring anaerobic capacity.
\end{abstract}

Key words: Reliability, shuttle run, basketball, high school.

\section{Introduction}

The game of basketball requires endurance, speed, agility, and power $[1,2]$ with some feeling that anaerobic capacity is the most important physical attribute [3]. Basketball coaches who pay attention to the strength and conditioning aspects of preparing their athletes know that they need to develop the anaerobic capacity of their athletes in order to compete at the highest levels. In order to assess the outcomes of strength and conditioning programs coaches must have access to valid and reliable tests.

The lab based Wingate cycle test in considered the "Gold Standard" test with regards to assessing anaerobic capacity and has been previously described [4]. However, access to facilities, qualified personnel and the time required to conduct the Wingate cycle test are not possible/practical for most High School athletic teams (an issue compounded for girls athletic teams).

Given the limitations with regards to utilizing the

Corresponding author: Mark DeBeliso, Ph.D., research fields: orthopedic biomechanics, mechanics and metabolics of sport movements and work-tasks, strength training for all walks of life, and master's athletes. E-mail: markdebeliso@suu.edu.
Wingate cycle test, many practitioners have looked to practical and meaningful field tests. A commonly used field test to assess anaerobic capacity is the 300-yard shuttle run and is recognized as such by the NSCA (National Strength and Conditioning Association) [5-7].

While the 300-yard shuttle run test is widely used to assess anaerobic capacity, there is a paucity of research with regards to documenting the reliability of the 300 -yard shuttle run in various populations. Hence, the purpose of this study was to determine the reliability of the 300-yard shuttle run in a population of High School girls basketball players.

\section{Methods}

\subsection{Participants}

A convenience sample of High School girls basketball players were recruited and later volunteered for the study. Permission to conduct the study was obtained through an University Institutional Review Board. Each participant and parent gave written consent before engaging in the study. 


\subsection{Procedures}

There were two testing sessions that included a warmup period consisting of jumping jacks, butt kickers, walking leg hugs, straight leg walking high kicks, kareoka, walking lunges with a twist, and body weight squats ( $\approx 8$ minutes). Each testing session was performed on separate days and included the participants completing two trials of the 300-yard shuttle run as described by Gillam \& Marks [7]. Each trial was separated by a 5-min rest period [7]. Two pairs of cones were used to establish two lines set apart by 25-yard in the High School gymnasium. The participants performed 6 full trips of running at maximum speed back and forth between the lines established by the pairs of cones (Fig. 1). The trials were recorded by hand with stop watches to the 10th of a second. The trials were separated by a 5 min rest period as described by Gillam \& Marks [7]. The same timers were used for all trials. One timer measured the shuttle run times and another was the rest timer. The testing sessions were conducted during the basketball offseason. The participants had previous experience performing the shuttle run.

\subsection{Statistical Analysis}

A combination of statistical approaches were used to determine the test-retest reliability of the 300-yard shuttle run (trial 1 vs. trial 2). Interclass (r) and intraclass reliability coefficients (ICC) were calculated. The standard error of the measure $\left(\mathrm{SE}_{\mathrm{m}}\right)$ was also calculated. Scatter and Bland-Altman plots were constructed in order to explore uniformity of error. Trial data was Log-transformed in order to assess typical error as expressed as a coefficient of variation percent (CV\%). Further, 90\% confidence limits were calculated for the aforementioned reliability statistics. Statistics were calculated in Excel with a spreadsheet prepared by Hopkins [8].

\section{Results}

Thirty nine High School girls basketball players participated in the study. The average age, height, and body mass of the participants is presented in Table 1 . All of the participants completed both test sessions of the shuttle run without incident. One participant's data was corrupted and the associated trial scores were omitted from the reliability analysis.

The reliability analysis is based on two sessions of test re-test scores $(n=38)$, hence a total of 76 pairs of shuttle run scores. The mean of trial 1 and trial 2 scores from both sessions were $66.2 \pm 4.9$ and $69.1 \pm 6.0$ seconds, respectively, with a mean difference between trials of $2.9 \pm 3.2$ seconds. Fig. 2 is a scatter plot of trial 1 and 2 shuttle run scores, while Fig. 3 is a Bland-Altman plot comparing the trial average scores versus the difference scores. The scatter plot suggests a linear relationship between the trial scores. The Bland-Altman plot demonstrates that only 4 of 76 data pairs $(\approx 5 \%$ ) exceeded the $95 \%$ limits of agreement, which is considered reliable [9]. However, the plot also suggested a bias of slower times during trial 2. Both plots suggested heteroscedasticity or non-uniform error. The variability between trial 1 and 2 scores appeared to increase with increasing shuttle run times. Of the 76 pairs of shuttle run scores, 61 were slower during trial 2 ,

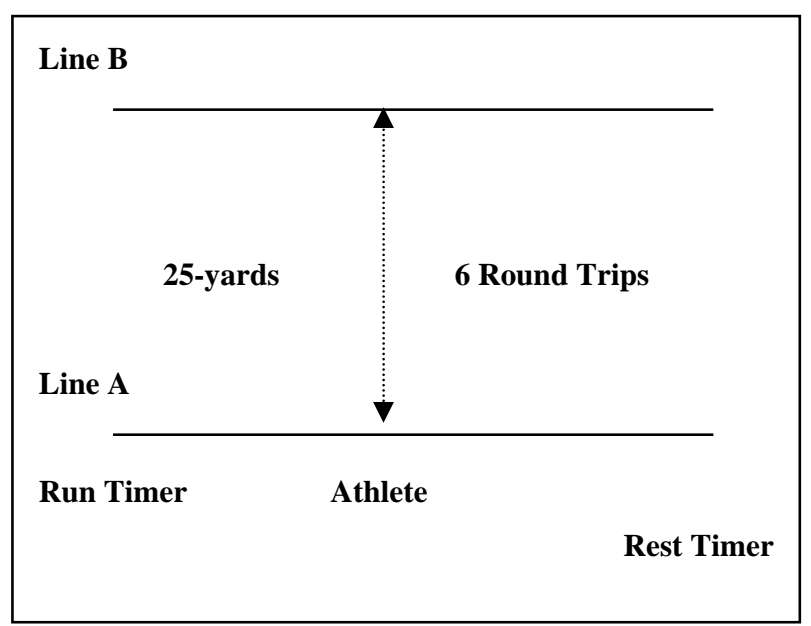

Fig. 1 Shuttle Run Course (6 round trips: $12 \times 25=300$ yards).

Table 1 Participant Descriptive Information.

\begin{tabular}{lll}
\hline Age (years) & Height $(\mathrm{cm})$ & Mass $(\mathrm{kg})$ \\
\hline $16.1 \pm 0.9$ & $163.4 \pm 38.3$ & $60.4 \pm 17.3$ \\
\hline
\end{tabular}

High School Girls Basketball players, $N=38$ (mean \pm sd). 


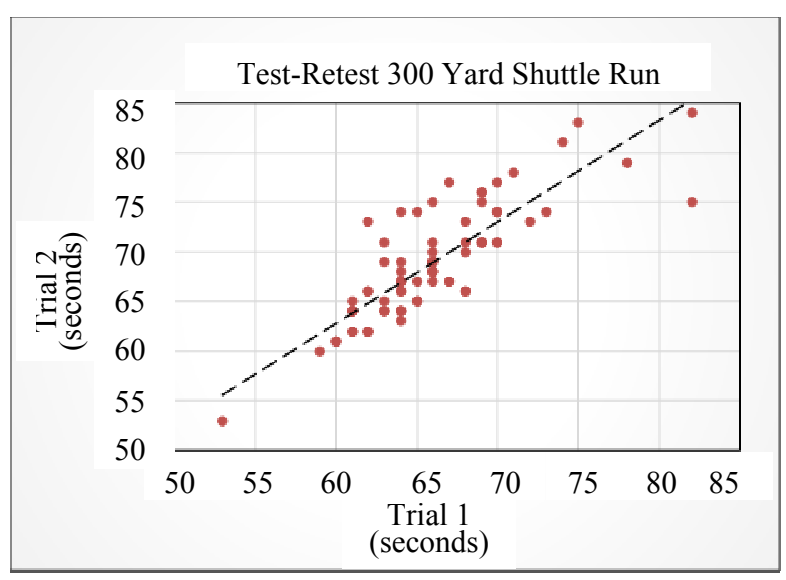

Fig. 2 Scatter plot of Trial 1 and Trial 2 shuttle run scores.

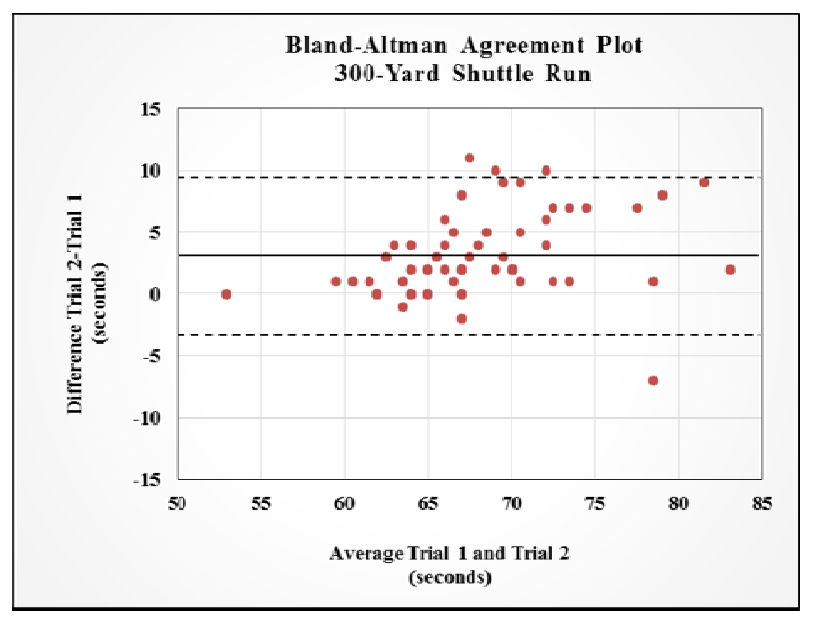

Fig. 3 Bland-Altman plot of shuttle run Trial scores.

Table 2 300-yard shuttle run reliability statistics.

\begin{tabular}{llll}
\hline Statistic & & $\begin{array}{l}\text { Upper } \\
\text { Limit }\end{array}$ & $\begin{array}{l}\text { Lower } \\
\text { Limit }\end{array}$ \\
\hline$\Delta$ Means (sec) & $2.9 \pm 3.2$ & 3.5 & 2.3 \\
$\mathrm{r}$ & 0.84 & 0.89 & 0.78 \\
$\mathrm{ICC}$ & 0.83 & 0.88 & 0.76 \\
Typical Error $(\mathrm{CV} \%)^{*}$ & 3.2 & 3.7 & 2.8 \\
$\mathrm{SE}_{\mathrm{m}}$ & 2.2 & 2.6 & 1.9 \\
\hline
\end{tabular}

$90 \%$ confidence limits for selected reliability statistics. *Typical error expressed as a CV\% based on Log-transformed data. SEm: standard error of the measure; r: Pearson correlation coefficient; ICC: Intraclass correlation coefficient.

eleven scores were unchanged between trials, and 4 were faster during trial 2.

Table 2 provides the reliability statistics with $90 \%$ confidence limits $\left(\mathrm{U}_{\mathrm{L}}, \mathrm{L}_{\mathrm{L}}\right)$. The intraclass reliability coefficient was ICC $=0.83(0.88,0.76)$, which is considered "average acceptable" [10]. The interclass reliability coefficient was $r=0.84(0.89,0.78)$, which is considered high [11]. The standard error of the measure was $\mathrm{SE}_{\mathrm{m}}=2.2(2.6,1.9)$ seconds. The trial data exhibited non-uniform error, as such the data was Log-transformed as suggested by Hopkins [12] in order to quantify typical error. The typical error as expressed as a coefficient of variation was $\mathrm{CV} \%=3.2(3.7,2.8)$ percent.

\section{Discussion}

The purpose of this study was to determine the reliability of the 300-yard shuttle run in a population of High School girls basketball players. The 300-yard trial measures collected during the current study compare favorably with previous studies [13-15]. Hoffman [13] presents percentile ranks for various fitness tests including the 300-yard shuttle run for NCAA (National Collegiate Athletic Association) women's basketball. The mean of the trial 1 scores in the current study $(66.2$ sec) would rank in the 30-40th percentile (66.8-65.9 sec) of NCAA women's basketball. Sporis et al. [15] collected 300-yard shuttle run scores in a population of elite women soccer players aged 18-19 years. The soccer player's 300-yard shuttle run scores $(66.5 \pm 4.2$ sec) were near identical to those collected during the first trial of the current study; although, it might have been expected that elite women soccer players would have been faster than the High School basketball players. Schaal [14] collected performance tests measures of High School girls volleyball players including two trials of the 300-yard shuttle. The mean of the 300-yard shuttle run scores (trial $1 \& 2$ ) collected by Schaal [14] was $70.0 \pm 6.9$ seconds.

The interclass reliability coefficient in the current study $(r=0.84)$ was identical to that reported in the aforementioned study by Schaal [14] $(r=0.84)$. Unfortunately, Sporis et al. [15] did not report an interclass correlation coefficient that we could compare to the current study. Further, if should be noted that the lower limit of the $90 \%$ confidence interval for the interclass reliability coefficient in the current study was $\left(\mathrm{L}_{\mathrm{L}}=0.78\right)$, which is considered to be moderate [11]. 
The intraclass reliability coefficient in the current study was $(\mathrm{ICC}=0.83$ ). The study by Schaal [14] did not report an ICC for the 300-yard shuttle run scores. Likewise, Sporis et al. [15] did not report a specific ICC for the 300-yard shuttle run scores but eluded to the ICC being in the range of $0.78-0.88$. However, it should be noted that the lower limit of the $90 \%$ confidence interval for the intraclass reliability coefficient in the current study is $\left(\mathrm{ICC} \mathrm{L}_{\mathrm{L}}=0.78\right)$, which is considered below average acceptable [10].

The $\mathrm{SE}_{\mathrm{m}}$ (standard error of measure) is a measure of absolute reliability [16]. The $\mathrm{SE}_{\mathrm{m}}$ in the current study suggests that when measuring the 300-yard shuttle run in this population that the true score is within \pm 2.2 seconds of the measured score ( $68 \%$ confidence). The implication here is that if a coach is attempting to use the 300-yard shuttle run as a measure of change in anaerobic capacity then the score change must exceed 2.2 seconds or it could likely be that the change in scores recorded was simply measurement error. It should also be noted that when scores reflect non-uniform error (as is the case in the current study), the $\mathrm{SE}_{\mathrm{m}}$ is biased and likely over estimates error in the lower scores and underestimates the error in the higher scores.

In order to correct for non-uniformity of error Hopkins [12] suggests Log-transforming the scores and expressing the error as "typical error" or a CV\%. The CV\% with $90 \%$ confidence limits represents $(\mathrm{CV} \%=$ 3.2: 3.7, 2.8) a non-bias measure of error that should be used as a bench mark when attempting to measure change in the 300-yard shuttle run scores in this population.

The second trials were much slower than the first trials in this study $(2.9 \pm 3.2$ seconds $)$. Significantly slower trial times were not reported in the Schaal study [14] and Sporis et al. [15] only reported the average of the two trial times. It is possible that the slower second trial times noted in the current study were due to fatigue, lack of motivation, or both.

If fatigue played a role in the slower second trial times, it may make sense to implement a rest period between trials beyond the suggested 5 minutes. Moore and Murphy [17] conducted two trials of the 300 meter shuttle run separated by 48 hours and reported a $\mathrm{SE}_{\mathrm{m}}=$ \pm 0.65 seconds (also known as the technical error of measurement $\mathrm{TE}_{\mathrm{m}}$ ). Given the extremely low $\mathrm{SE}_{\mathrm{m}}$ reported by Moore and Murphy [17], it would appear that fatigue may play a role in lower second trial scores when the second trial is proceeded by a rest period that is only 5 minutes in duration.

Another point worthy of discussing has to do with the actual value of collecting a second trial of shuttle run scores. Generally speaking, individuals typically have better scores during subsequent repeated trials of a physical test due to some type of learning. If that's not the case with the second trial of shuttle run scores (as is the case in this study), then why conduct two trials if one is looking for max performance? Is it possible that you only need one trial of the shuttle run to establish anaerobic capacity in this population? Further, if participants know there is only one trial, they may not hold back, knowing there is not another lactic acid burn on the way during the would-be second trial of the shuttle run.

So a very beneficial outcome of the current study may be that coaches and the athletes need only conduct one all-out effort of a single trial of the shuttle run in order to establish the athlete's anaerobic capacity. With that said, conducting two trials may be still of value with regards to providing insight into the recovery capacity of an individual from the first shuttle run trial.

\section{Conclusions}

Within the parameters of this study, the 300-yard shuttle run protocol demonstrated an acceptable level of reliability and should be considered a reliable test for measuring anaerobic capacity in High School girls basketball players. The 300-yard shuttle run is an easily administered useful measure of anaerobic capacity and the authors recommend its use to physical educators and youth sport coaches who work with this 
population.

\section{References}

[1] Siegler, J., Gaskill, S., and Ruby, B. 2003. "Changes Evaluated in Soccer-Specific Power Endurance either with or without a 10-Week, in-Season, Intermittent, High-Intensity Training Protocol." Journal of Strength and Conditioning Research 17 (2): 379-87.

[2] Balčiūnas, M., Stonkus, S., Abrantes, C., and Sampaio, J. 2006. "Long Term Effects of Different Training Modalities on Power, Speed, Skill and Anaerobic Capacity in Young Male Basketball Players." Journal of Sports Science \& Medicine 5 (1): 163-70.

[3] Apostolidis, N., Nassis, G. P., Bolatoglou, T., and Geladas, N. D. 2004. "Physiological and Technical Characteristics of Elite Young Basketball Players." Journal of Sports Medicine and Physical Fitness 44 (2): 157-63.

[4] Bar-Or, O. 1987. "The Wingate Anaerobic Test: An Update of Methodology, Validity and Reliability." Sport Medicine 4 (6): 381-94.

[5] Baechle, T. R., and Earle, R. W. 2008. Essentials of Strength Training and Conditioning (3rd Ed.). Champaign, IL: Human Kinetics.

[6] Jones, A. 1991. "300-yard Shuttle Run.” National Strength \& Conditioning Association Journal 13 (2): 56.

[7] Gillam, G. M., and Marks, M. 1983. "300-yard Shuttle Run." National Strength \& Conditioning Association Journal 5 (5): 46.

[8] Hopkins, W. G. 2007. "Understanding Statistics by Using Spreadsheets to Generate and Analyze Samples."
Sportscience 11: 23-36.

[9] Bland, J. M., and Altman, D. G. 1986. "Statistical Methods for Assessing Agreement between Two Methods of Clinical Measurement." Lancet 8 (8476): 307-10.

[10] Baumgartner, T. A., Jackson, A. S., Mahar, M. T., and Rowe, D. A. 2007. Measurements for Evaluation in Physical Education and Exercise Science (8th Ed.). New York, NY: McGraw Hill.

[11] Safrit, M. J., and Wood, T. M. 1995. Introduction to Measurement in Physical Education and Exercise (3rd Ed.). St. Louis, MO: Mosby.

[12] Hopkins, W. G. 2013. A New View of Statistics. Internet Society for Sport Science. Accessed September 30, 2015. http://www.sportsci.org/resource/stats/.

[13] Hoffman, J. 2006. Norms for Fitness, Performance, and Health. Champaign, IL: Human Kinetics.

[14] Schaal, M. L. 2011. "Physiologic Performance Test Differences by Competition Level and Player Position in Female Volleyball Athletes." Masters Thesis, Boise State University. Boise, ID.

[15] Sporiš, G., Jovanovic, M., Krakan, I., and Fiorentini, F. 2011. "Effects of Strength Training on Aerobic and Anaerobic Power in Female Soccer Players." Sport Science 4 (2): 32-7.

[16] Bruton, A, Conway, J. H., and Holgate, S. T. 2000. "Reliability: What Is It and how Is It Measured?" Physiotherapy 86 (2): 94-9.

[17] Moore, A., and Murphy, A. 2003. "Development of an Anaerobic Capacity Test for Field Sport Athletes." Journal of Science and Medicine in Sport 6 (3): 275-84. 